\title{
Qualidade de vida em cem alunos do curso de Medicina de Sorocaba - PUC/SP
}

\author{
Quality of life among 100 medical students at \\ the Catholic University in Sorocaba, São Paulo
}

PALAVRAS-CHAVE

- Qualidade de Vida.

- Estudantes de Medicina

- Consumo de Bebidas Alcoólicas

- Tabagismo
KEY WORDS

- Quality of life

- Students, Medical

- Alcohol Drinking

- Smoking
Recebido em: 27/10/2008 Reencaminhado em: 11/04/2009 Aprovado em: 19/07/2009

REVISTA BRASILEIRA DE EDUCAÇÃO MÉDICA

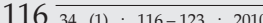

João Carlos Ramos-Dias

Milena Carvalho Libardi ${ }^{\mathrm{I}}$

Carina Maria Zillo ${ }^{\mathrm{I}}$

Mamy Honda Igarashi ${ }^{\mathrm{I}}$

Maria Helena Senger

\begin{abstract}
R E S U M O
Poucos estudos analisaram a qualidade de vida $(\mathrm{QV})$ em populações específicas, como os profissionais da área de saúde. Avaliamos a QV por meio do Whoqol-bref em estudantes do primeiro $(n=50)$ e sexto $(n=50)$ anos do curso de Medicina. A pontuação média dos alunos do primeiro e sexto anos (escala de 0-100) em cada domínio foi, respectivamente, de 77,9 e 76,8 (físico), 71,1 e 72,6 (psicológico), 70,2 e 77,8 (relações sociais) e 67,7 e 70,1 (ambiental). Houve diferença estatística entre os dois grupos apenas no domínio das relações sociais $(\mathrm{p}<0,05)$. Nossos resultados demonstraram pontuações altas em todos os domínios do Whoqol-bref, indicando uma boa QV nos grupos estudados. Os alunos do primeiro ano estão se adaptando à nova vida acadêmica, o que provavelmente contribuiu para sua menor pontuação no domínio das relações sociais. Aproximadamente um terço dos alunos dos dois grupos pontuou dentro do primeiro quartil em três ou em todos os domínios, indicando aqueles com pior QV. Isto sugere que estes indivíduos devem receber maior atenção, para prevenir o risco de burnout descrito na literatura entre estudantes de Medicina.
\end{abstract}

\section{A B S T R A C T}

Few studies have evaluated quality of life (QOL) in specific populations such as health professionals. We evaluated QOL using the Whoqol-bref in first-year $(n=50)$ and sixth-year $(n=50)$ medical students. The mean scores for first and sixth-year students (on a scale from 0 to 100) in each domain were, respectively, 77.9 and 76.8 (physical), 71.1 and 72.6 (psychological), 70.2 and 77.8 (social relations), and 67.7 and 70.1 (environmental). There was only a statistically significant difference between the two groups in the social relations domain $(p<0.05)$. Our results showed high scores in all the domains of Whoqol-bref, indicating good QOL in the target groups. First-year students are adapting to their new academic life, which probably contributes to their lower scores in the social relations domain. Approximately one-third of the students in the two groups scored within the first quartile in three or all of the domains, indicating those with worse QOL. This suggests that these individuals should receive more attention in order to prevent the risk of burnout described in the literature for medical students.

${ }^{I}$ Pontifícia Universidade Católica de São Paulo, Sorocaba, SP, Brasil. 


\section{INTRODUÇÃO}

A Organização Mundial da Saúde $(\mathrm{OMS})^{1}$ define qualidade de vida $(\mathrm{QV})$ como "a percepção do indivíduo de sua posição na vida, no contexto da cultura e sistema de valores nos quais vive e em relação aos seus objetivos, expectativas, padrões e preocupações". Essa definição inclui seis domínios principais: saúde física, estado psicológico, níveis de independência, relacionamento social, características ambientais e padrão espiritual.

O termo QV remete a interpretações individuais diferenciadas, o que dificulta sua avaliação, pois cada pessoa pode enfatizar um de seus domínios. Para uns, a QV se restringe à saúde física, já para outros, apenas ao relacionamento social ${ }^{2}$.

$\mathrm{Na}$ área da saúde, o conceito de $\mathrm{QV}$ está relacionado à saúde física, psíquica e mental do indivíduo na presença de uma doença, de um tratamento ou de um trauma. O enfoque habitualmente conferido pelos profissionais da saúde ao termo QV enfatiza mais os aspectos biológicos e orgânicos, com os quais têm maior convivência $2^{2-4}$. Embora o profissional da área da saúde, especificamente o médico, seja o responsável pelas atividades de promoção à saúde de forma ampla e integral, incluindo não apenas o aspecto físico do indivíduo, mas também o psicológico e sua inserção no ambiente em que vive, sabe-se que estes profissionais nem sempre aplicam estes conceitos em benefício próprio, conforme resultados de estudos que abordam o consumo de álcool e o tabagismo no meio médico ${ }^{5-8}$.

Um grupo especial pertencente à área da saúde é composto pelos estudantes de Medicina, já que serão os futuros responsáveis por ações ativadoras de uma melhor QV. Como ainda estão em formação, nosso objetivo foi analisar se a futura classe médica se preocupa com a própria QV. Avaliamos estes indivíduos em dois momentos distintos do curso de Medicina, representados pelos alunos do primeiro e do sexto anos.

\section{MÉTODOS}

Este estudo foi desenvolvido com alunos do curso de Medicina da Faculdade de Ciências Médicas de Sorocaba - SP, após aprovação pelo Comitê de Ética local. Todos os participantes assinaram o consentimento informado antes do início da pesquisa. Avaliamos 50 alunos do primeiro ano e 50 alunos do sexto ano do referido curso, cujas classes tinham 100 alunos, aproximadamente. No cálculo do tamanho da amostra foram utilizados dados da literatura referente ao questionário Whoqol-bref sobre qualidade de vida numa população sadia ${ }^{9}$. O maior desvio-padrão (DP) de um dos domínios do questionário (relações sociais) foi inserido no programa Stata 8.0, e o resultado obtido foi de uma amostra de 48 pessoas. Como o maior desvio-padrão é o mais desfavorável, a amostra calculada pelo programa é significativa.
Todos os alunos do primeiro e do sexto anos foram convidados a participar do estudo, e a coleta de dados foi encerrada com os primeiros 50 alunos de cada ano que atenderam ao convite.

A avaliação da QV de cada participante do estudo foi realizada por meio de um questionário específico para este fim, o Whoqol-bref, proposto pela OMS, traduzido e validado em nosso meio por Fleck et al. ${ }^{10}$. Este questionário aborda o tema QV por meio dos seguintes domínios: físico, psicológico, relações sociais e ambiental. O questionário é composto por 26 questões. Duas questões são gerais (sobre QV e saúde), e as outras 24 representam os domínios acima citados. Os resultados foram analisados com o programa estatístico SPSS 11.0.1. Para cada resposta é atribuída uma pontuação, e o programa fornece o somatório de cada indivíduo, sendo que esta pontuação pode variar de 0 a 100. Quanto maior a pontuação, melhor a QV do indivíduo estudado. Também analisamos a distribuição dos valores em quartis e consideramos como as piores pontuações, dentro do grupo estudado, as pertencentes ao primeiro quartil (25\%).

Para a análise de hábitos e vícios, coletamos dados relacionados a tabagismo e consumo de bebidas alcoólicas em todo o grupo estudado. Para o tabagismo, avaliamos o número de cigarros consumidos por dia, a duração do hábito de fumar e se existia o desejo de abandonar o fumo. A investigação do consumo de bebidas alcoólicas incluiu perguntas sobre tipo, frequência e tempo de uso. Também utilizamos o questionário Cage (cut down/annoyed/guilty/eye-opener), instrumento aplicado em serviços de triagem para determinar se um indivíduo faz uso de álcool de maneira nociva ou de risco. Este questionário, de fácil aplicação, consiste em quatro questões que comportam as respostas sim ou não. Serve para identificar os consumidores de risco e não para diagnosticar a dependência, sendo que os indivíduos com teste positivo (duas ou mais respostas sim) são aqueles aos quais se deve propor uma intervenção ${ }^{11}$.

Também incluímos no estudo uma avaliação socioeconômica de todos os participantes. Utilizamos para este fim um questionário específico, o Critério de Classificação Econômica Brasil, com perguntas sobre renda familiar, moradia, posses e grau de instrução do chefe da famíliaa ${ }^{12}$.

Os resultados são apresentados como média \pm DP. Para a análise estatística dos dados obtidos, utilizamos o programa BioEstat 2.0. Na comparação da idade entre os grupos, utilizamos o teste Anova. O teste de Mann-Whitney foi utilizado para comparar os escores obtidos nos diferentes domínios do Whoqol-bref entre os alunos do primeiro e do sexto anos.

Foi considerado significante o valor de $\mathrm{p}<0,05$. 


\section{RESULTADOS}

Entre os alunos do primeiro ano ( 30 homens e 20 mulheres), a idade média verificada foi de 20,5 $\pm 1,8$ anos (variação de 18 a 27 anos). Nos alunos do sexto ano (19 homens e 31 mulheres), a idade média foi de 24,4 \pm 1,3 anos (variação de 22 a 28 anos). Houve diferença estatística significante entre as idades quando comparamos os dois grupos $(\mathrm{p}<0,01)$.

Em relação ao estado civil, todos os indivíduos avaliados eram solteiros.

A maioria dos alunos do primeiro ano é natural do Estado de São Paulo ( $\mathrm{n}=47 ; 94 \%$ ), sendo oito da cidade de Sorocaba. Dois alunos são de outros Estados (Rio de Janeiro e Paraná), e um é estrangeiro (Equador). Quanto aos antecedentes mórbidos pessoais (AMP), a maioria (92\%) não apresentou relato positivo, e apenas quatro alunos registraram AMP de asma $(n=1)$, rinite $(n=1)$, amidalectomia $(n=1)$ e apendicectomia $(n=1)$.

Quanto à naturalidade dos alunos do sexto ano, 46 (92\%) são do Estado de São Paulo (5 de Sorocaba). Três indivíduos são de outros Estados (Bahia, Paraná e Minas Gerais), e um aluno é estrangeiro (Estados Unidos). Os AMP não foram dignos de nota em 29 alunos (58\%). No restante do grupo, houve relatos de asma $(n=6)$, rinite $(n=7)$, hipertensão $(n=1)$, deficiência de lactase $(\mathrm{n}=1)$, nefrolitíase $(\mathrm{n}=2)$, dislipidemia $(\mathrm{n}=1)$, apendicite $(\mathrm{n}$ $=1)$, bronquite $(n=1)$, síndrome dos ovários policísticos (SOP) (n $=2$ ) e esofagite de refluxo $(n=1)$. Alguns alunos apresentaram mais de um AMP.

\section{Qualidade de vida}

A pontuação média dos alunos do primeiro ano nos 4 domínios do Whoqol-bref foi de 77,9 9 9,8 (físico), 71,1 \pm 10,9 (psicoló- gico), 70,2 \pm 17,4 (relações sociais) e 67,7 $\pm 11,7$ (ambiental), lembrando que os escores individuais poderiam variar de 0 a 100 (Gráfico 1). Analisando a distribuição dos valores de acordo com os quartis, observamos que 11 alunos (22\%) tiveram menores pontuações (igual ou inferior ao quartil 25) em pelo menos $3 \mathrm{dos}$ domínios, e 7 outros alunos (14\%) nos 4 domínios avaliados.

Entre os alunos do sexto ano a pontuação média observada em cada domínio foi de 76,8 \pm 12,6 (físico), 72,6 $6 \pm 11,1$ (psicológico), 77,8 $8 \pm 14,5$ (relações sociais) e 70,1 $\pm 11,9$ (ambiental) (Gráfico 1). Neste grupo, 8 alunos (16\%) pontuaram dentro do primeiro quartil em pelo menos 3 domínios, e outros 11 alunos (22\%) em todos os domínios.

A comparação entre os dois grupos mostrou diferença estatística apenas no domínio das relações sociais $(p<0,05)$.

O valor médio e a variação da pontuação obtida em cada um dos domínios estão representados na Tabela 1. A variação é apresentada entre parênteses.

Além da avaliação dos domínios acima citados, o questionário sobre QV contava com duas questões gerais. A primeira delas avaliava como o indivíduo considerava sua QV. Em sua resposta, a maioria dos alunos, tanto do primeiro ano (86\%) como do sexto ano (88\%), a considerou "boa" ou "muito boa". Cinco alunos do primeiro ano (10\%) e 6 do sexto ano (12\%) consideraram sua qualidade de vida "nem ruim, nem boa", enquanto apenas 2 alunos do primeiro ano (4\%) responderam que sua QV era "ruim".

A segunda questão de âmbito geral avaliava o quanto o indivíduo estava "satisfeito com sua saúde". Nesta questão, $74 \%$ ( $n=37)$ dos alunos tanto do primeiro ano como do sexto ano responderam que estavam "satisfeitos" ou "muito satisfeitos". "Nem satisfeito,

Gráfico 1

Valores médios $( \pm \mathrm{DP})$ da pontuação nos diferentes domínios do Whoqol-bref entre alunos do primeiro ano $(\mathrm{n}=$

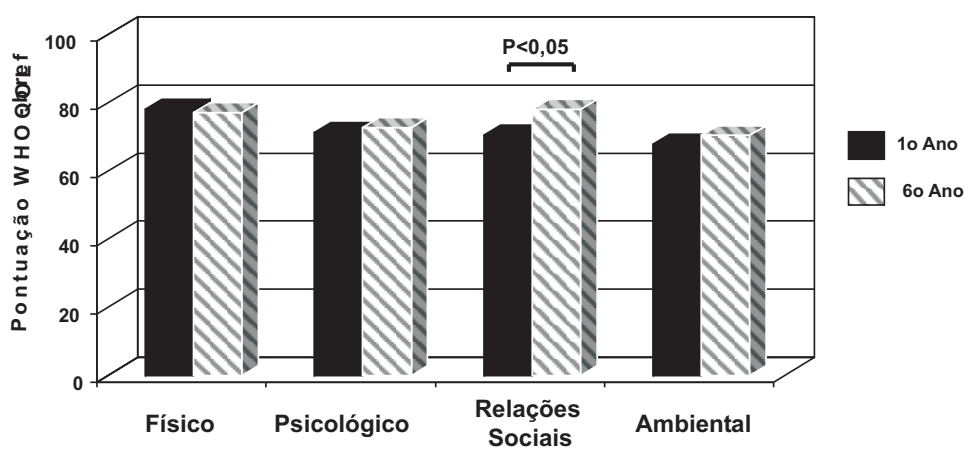


nem insatisfeito" foi a resposta de 10 alunos do primeiro ano (20\%) e 9 alunos do sexto ano (18\%). Três alunos do primeiro (6\%) e 4 do sexto ano $(8 \%)$ mostraram-se "insatisfeitos" com sua saúde. sumidores, 4 eram mulheres e 3 homens. No sexto ano, $82 \%$ dos alunos $(n=41)$ referiram consumir bebidas alcoólicas. Neste grupo, as bebidas mais mencionadas também foram cerveja $(\mathrm{n}=$

Tabela 1

Valores médios ( \pm DP) da pontuação nos diferentes domínios do questionário de Qualidade de Vida Whoquol-bref, entre alunos do primeiro ano $(n=50)$ e do sexto ano $(n=50)$ do curso de Medicina

\begin{tabular}{l|c|c|c}
\hline Domínio & $1^{\circ}$ Ano & $6^{\circ}$ Ano & Título??? \\
\hline \multirow{2}{*}{ Físico } & $77,9 \pm 9,8$ & $76,8 \pm 12,6$ & $\mathrm{p}=\mathrm{NS}$ \\
& $(53,6-96,4)$ & $(46,4-100)$ & $\mathrm{p}=\mathrm{NS}$ \\
& & $72,6 \pm 11,1$ & $(45,8-95,8)$ \\
Psicológico & $71,1 \pm 10,9$ & & $\mathrm{p}<0,05$ \\
& $(37,5-91,7)$ & $77,8 \pm 14,5$ & $(33,3-100)$ \\
Relações sociais & & & $\mathrm{p}$ \\
& $(16,7-83,3)$ & $70,1 \pm 11,9$ & $(40,6-100)$ \\
\hline
\end{tabular}

\section{Tabagismo e consumo de bebidas alcoólicas}

Apenas quatro alunos (8\%) do primeiro ano tinham o hábito de fumar, sendo que 2 deles se sentiam motivados a parar. O número de cigarros/dia foi de $3(n=2), 6(n=1)$ ou $10(n=1)$, e o tempo de consumo de 2 a 3 anos. Dois outros alunos desta série abandonaram o cigarro havia um e seis meses, respectivamente. Entre os alunos do sexto ano encontramos somente 2 tabagistas (4\%), sendo um ocasional e o outro consumidor de 10 cigarros/dia. O tempo de consumo foi de 10 e 7 anos, respectivamente. O consumidor ocasional sente-se motivado a parar de fumar. Entre os alunos do sexto ano não houve ex-fumantes.

O consumo de álcool foi referido por $86 \%$ dos alunos do primeiro ano $(n=43)$, e todos tiveram o Cage negativo. As bebidas mais mencionadas foram cerveja $(n=31)$, vodca $(n=24)$, vinho $(n=24)$ e uísque $(n=9)$. Sete não especificaram o tipo de bebida consumida. Na maior parte do grupo, o uso é ocasional (41,9\%) ou de 1 a 2 vezes por semana (44,2\%) e, no restante, de 3 a 4 vezes por semana ( $n=6 ; 13,9 \%)$. O tempo médio desde o início do consumo foi de 3,3 $\pm 1,4$ anos (variação: 1 a 7 anos). Entre os não con-
$31)$, vodca $(n=19)$, vinho $(n=17)$ e uísque $(n=8)$. Houve ainda menção ao consumo de pinga $(n=1)$, saquê $(n=1)$ e chope de vinho $(n=3)$. Três alunos não mencionaram o tipo de bebida consumida. A grande maioria faz uso de álcool de forma ocasional (61\%) ou de 1 a 2 vezes por semana (34,2\%). Apenas 1 consome bebida de 3 a 4 vezes por semana (cerveja). Os sextanistas referem consumir bebidas alcoólicas havia um tempo médio de 7,2 \pm 2,3 anos, com variação de 3 a 10 anos. Em 1 homem, o Cage foi positivo, e o consumo relatado por ele é de cerveja, vinho e uísque, de 1 a 2 vezes por semana. Entre os não consumidores observamos 8 mulheres e 1 homem.

\section{Avaliação socioeconômica}

De acordo com o Critério de Classificação Econômica Brasil, que enfatiza a estimativa do poder de compra das pessoas e famílias urbanas, a maioria dos alunos foi classificada economicamente nas classes A1 e A2 (66\% dos alunos do primeiro ano e $88 \%$ dos alunos do sexto ano). O restante dos alunos do sexto ano pertence às classes B1 e B2, enquanto no primeiro ano, além 
destas classes $(n=10,20 \%)$, também existem alunos das classes $\mathrm{C}(\mathrm{n}=4 ; 8 \%)$ e $\mathrm{D}(\mathrm{n}=1 ; 2 \%)$. Apenas um aluno de cada ano não respondeu a este item. A Tabela 2 mostra a distribuição da renda familiar entre os alunos avaliados. Os valores são apresentados em percentagem (\%) e números absolutos. sendo o Whoqol apropriado tanto para populações doentes, como para indivíduos saudáveis ${ }^{2,9,17}$. Optamos pelo questionário reduzido, por ser de mais fácil aplicação (26 questões, contra 100 questões do questionário completo), além de abranger todos os pontos de interesse de nosso estudo e já estar validado em nosso meio m.10. $^{9}$

Tabela 2

Distribuição da renda familiar entre os alunos do primeiro ano $(n=50)$ e do sexto ano $(n=50)$ do curso de Medicina de Sorocaba

\begin{tabular}{lcc}
\hline Renda familiar $(\mathrm{R} \$)$ & $1^{\circ}$ Ano $(\mathrm{n}=50)$ & $6^{\circ}$ Ano $(\mathrm{n}=50)$ \\
\hline$\geq 7.500,00$ & $58 \%(\mathrm{n}=29)$ & $62 \%(\mathrm{n}=31)$ \\
$4.500,00$ a $7.500,00$ & $20 \%(\mathrm{n}=10)$ & $24 \%(\mathrm{n}=12)$ \\
$2.800,00$ a $4.500,00$ & $10 \%(\mathrm{n}=5)$ & $4 \%(\mathrm{n}=2)$ \\
$1.650,00$ a $2.800,00$ & $6 \%(\mathrm{n}=3)$ & - \\
900,00 a $1.650,00$ & $2 \%(\mathrm{n}=1)$ & $8 \%(\mathrm{n}=4)$ \\
Não respondeu & $4 \%(\mathrm{n}=2)$ & \\
\hline
\end{tabular}

\section{DISCUSSÃO}

Avaliamos a QV em dois momentos distintos do curso de Medicina, representados pelos alunos do primeiro e do sexto anos. Nos dois grupos encontramos pontuações elevadas em todos os domínios do questionário utilizado (Whoqol-bref), indicando uma boa QV nestes indivíduos.

Poucos estudos em nosso meio utilizaram um instrumento validado para abordar QV em populações específicas, como profissionais de saúde ${ }^{13,14}$. Buscamos selecionar períodos do curso de Medicina em que os alunos estariam em estágios diferentes de sua aprendizagem e vivência. O primeiranista está se adaptando a um novo ambiente, já que a maioria não é procedente de Sorocaba. Já o sextanista está mais adaptado a esse ambiente e detém mais informações e consciência sobre seu estilo de vida. No entanto, o aluno do último ano da graduação em Medicina vivencia um período estressante, uma vez que tem em seu cotidiano os plantões, preocupações com os exames para a residência, maior contato com a relação entre vida e morte, e grandes decisões sobre a carreira ${ }^{15,16}$.

Avaliar QV é um processo complexo, pois envolve parâmetros subjetivos, como bem-estar, felicidade, amor, prazer e realização pessoal, além de medidas objetivas que refletem a satisfação das necessidades básicas, econômicas e sociais de determinada sociedade ${ }^{2}$. Existem vários métodos para analisar $\mathrm{QV}^{17}$,
Encontramos escores elevados em todos os domínios do Whoqol-bref, nas duas turmas estudadas. Nossos valores são semelhantes aos encontrados em outras populações sadias ${ }^{13}$ e maiores que os demonstrados em doentes e idosos ${ }^{18,19}$, sugerindo, portanto, boa QV nos grupos avaliados. Nas questões gerais, observamos que a maioria dos alunos também está satisfeita com sua QV e com sua saúde.

Quando comparamos os escores dos dois grupos de alunos, encontramos diferença apenas no domínio das relações sociais, que foi menor nos alunos do primeiro ano. A análise deste domínio envolve três perguntas que avaliam a satisfação do indivíduo quanto as suas relações pessoais (amigos, parentes, conhecidos e colegas), vida sexual e apoio que recebe dos amigos. Provavelmente, este índice mais baixo reflete uma fase de adaptação dos alunos do primeiro ano, pois a entrada na faculdade é um período de grandes mudanças, quando estes indivíduos se moldam a um novo "formato de vida". Como a maioria dos alunos do primeiro ano vem de outras cidades, eles têm que se ajustar a novos hábitos alimentares, horários e acomodações, muitos talvez completamente diferentes dos que tinham antes. Além disso, há necessidade de adequação emocional e acadêmica a uma "nova vida" longe da família e amigos. A maior pontuação dos alunos do sexto ano neste domínio demonstra, provavelmente, 
uma adaptação ao ambiente acadêmico ao longo da graduação. As diferenças também podem decorrer da constituição da amostra por voluntários, ainda que seu número tenha sido representativo de indivíduos submetidos às mesmas condições locais dentro da instituição.

Apesar da alta pontuação média em todos os domínios, chama a atenção o fato de que aproximadamente um terço dos indivíduos, tanto do primeiro como do sexto ano, teve escores iguais ou inferiores ao primeiro quartil (25\%) em pelo menos três ou nos quatro domínios avaliados. Estes alunos possivelmente necessitam receber maior atenção, já que podem ser considerados, dentro do grupo, aqueles com mais baixa QV. Os estudos de Parkerson et al. ${ }^{20}$ e Ball et al..$^{21}$ demonstram incremento da insatisfação em vários aspectos da vida em estudantes do primeiro ano do curso médico, incluindo aumento de sintomas depressivos. Além disso, outros estudos avaliaram a presença de sintomas de exaustão (burnout) entre estudantes de Medicina ${ }^{16,22,23}$, sugerindo também alta prevalência de estresse e ansiedade neste grupo, com alterações psicológicas maiores que na população geral ${ }^{16,24}$.

Embora a grande maioria dos jovens primeiro ou sextanistas tenha considerado sua QV como boa, aproximadamente um quarto deles em cada ano não se encontrava plenamente satisfeito com sua saúde. Este dado é intrigante, já que estamos lidando com uma população jovem, em sua grande maioria com alto poder aquisitivo e, teoricamente, com amplo acesso aos mecanismos de promoção à saúde.

Em relação ao tabagismo, observamos um número surpreendentemente pequeno de fumantes tanto no primeiro como no sexto ano. Embora este seja um fato bastante positivo se considerarmos os malefícios do uso do tabaco ${ }^{25}$, não podemos descartar um viés de amostra, pois nossos achados são contrários a outros estudos que mostram um consumo alto de tabaco entre estudantes do ensino médio e universitários em geral ${ }^{5,8,26-28}$.

O consumo de bebidas alcoólicas foi alto nos dois grupos avaliados e detectamos um bebedor de risco, embora a maioria dos alunos tenha relatado uso de álcool apenas ocasional ou de uma a duas vezes por semana. A alta prevalência no consumo de álcool é um fenômeno que vem sendo cada vez mais observado em alunos de Medicina, como também na classe médica ${ }^{5-8}$.

Ao avaliarmos a qualidade de vida dos alunos tabagistas e do bebedor de risco, verificamos que apenas um tabagista pontuou acima da média em todos os domínios. Os demais alunos apresentaram uma variabilidade de pontuação em relação ao valor médio de cada domínio que não justificou uma análise de correlação. $\mathrm{O}$ aluno considerado bebedor de risco apresentou uma pontuação próxima à média no domínio físico, abaixo do primeiro quartil no domínio ambiental e no terceiro quartil nos domínios psicológico e das relações sociais.

Quanto à avaliação socioeconômica, a maioria dos alunos do primeiro e do sexto ano foi classificada nas classes econômicas $\mathrm{A}$ e B, conforme esperado, já que nosso estudo foi efetuado numa escola privada, embora filantrópica. A presença de alunos do primeiro ano classificados nas classes econômicas C e Dé um aspecto bastante positivo e pode refletir a adesão da instituição ao Programa Universidade para Todos (ProUni).

Nosso estudo demonstrou que não existe diferença importante nos parâmetros avaliados quanto $\mathrm{QV}$ entre alunos do primeiro e sexto anos, embora o sextanista conviva com maior pressão, pela proximidade do término do curso, grande carga horária e estresse de plantões. Entretanto, um terço destes alunos foi classificado dentro do grupo como aqueles com pior QV (inferior ou igual ao primeiro quartil). Consideramos interessante que esta detecção seja mais precoce, a fim de permitir o acompanhamento destes alunos ao longo do curso. Isto pode ser particularmente importante durante os primeiros anos da vida acadêmica, como uma tentativa de reverter este quadro e para que este aluno chegue com melhor QV ao final da graduação. Está demonstrado que um suporte psicológico e educacional é efetivo em auxiliar o aluno a melhorar seus hábitos de vida, com a prática de hábitos saudáveis por meio da autoconsciência, autoestima e melhor conhecimento da sua situação ${ }^{21}$.

Ao concluir este trabalho, nos parece importante sua continuidade em busca do melhor conhecimento do perfil dos alunos do curso de Medicina. A proposta de reavaliar o grupo de alunos do primeiro ano, objeto desta pesquisa, quando estiverem no terceiro e sexto anos do curso está sendo viabilizada, uma vez que dados de literatura apontam um aumento da prevalência de exaustão (burnout) entre os estudantes ao longo do curso de Medicina ${ }^{22}$. Trata-se de uma população jovem, relacionada à área da saúde e pertencente, em sua maioria, às classes de maior recurso financeiro, na qual sempre deveria ser reforçada a consciência da prática de hábitos de vida saudáveis e suas consequências positivas sobre o organismo e QV, o que deverá se refletir em seu trabalho futuro como médicos e, principalmente, no aconselhamento de seus pacientes.

\section{REFERÊNCIAS}

1. WHOQOL Group. The World Health Organization Quality of Life assessment (WHOQOL): position paper from the World Health Organization. Soc Sci Med. 1995;41(10):1403-9.

2. Minayo MCS, Hartz ZMA, Buss PM. Quality of life and health: a necessary debate. Ciên Saúde Colet. 2000;5(1):7-18. 
3. Buss PM. Promoção da Saúde e Qualidade de Vida. Cien Saude Colet. 2000;5(1):163-77.

4. Segre M, Ferraz FC. O conceito de saúde. Rev Saúde Pública. 1997;31(5):538-42.

5. Barría ACR, Queiroz S, Nicastri S, Andrade AG. Comportamento do universitário da área de biológicas da Universidade de São Paulo. Rev Psiquiatr Clín [periódico na Internet]. 2000 [acesso em: 23 jul. 2008];27(4): [aproximadamente 12 p.]. Disponível em: http://www.hcnet.usp.br/ipq/revista/

6. Costa LFO, Alfani AC, Nro TOD, Chade MC, Greghl V, Tribist AL, et al. Comparação do uso de drogas entre acadêmicos do curso de medicina da Pontifícia Universidade Católica de São Paulo. Rev Fac Cienc Med. Sorocaba. 2004;6(1):7-14.

7. Kerr-Corrêa F, Andrade AG, Bassit AZ, Boccuto NMVF. Uso de álcool e drogas por estudantes de medicina da Unesp. Rev Bras Psiquiatr. 1999;21(2):95-100.

8. Souza FGM, Landim RM, Perdigão FB, Morais RM, Carneiro Filho BA. Consumo de drogas e desempenho acadêmico entre estudantes de medicina no Ceará. Rev Psiquiatr Clín [periódico na Internet]. 1999 [acesso em: 23 jul. 2008];26(4): [aproximadamente 11p.]. Disponível em: http:/ /www.hcnet.usp.br/ipq/revista/

9. Fleck MPA, Louzada S, Xavier M, Chachamovich E, Vieira G, Santos L et al. Aplicação da versão em português do instrumento abreviado de avaliação da qualidade de vida "WHOQOL - bref". Rev Saúde Pública [periódico na Internet]. 2000 [acesso em: 23 jul. 2008];34(2): aproximadamente 10p.]. Disponível em: www.fsp.usp.br/rsp

10. Fleck MPA, Fachel O, Louzada S, Xavier M, Chachamoviche E, Vieira G et al. Desenvolvimento da versão em português do instrumento de avaliação de qualidade de vida da Organização Mundial de Saúde (WHOQOL - 100). Rev Bras Psiquiatr. 1999;21(1):19-28.

11. Marques ACPR, Ribeiro M. Consenso. Consenso álcool diagnóstico do abuso e tratamento. Abuso e dependência do álcool [online]; 2002 [acesso em 23 jul. 2008]. Disponível em: http:/ / www.viverbem.fmb.unesp.br/consenso.asp

12. Associação Brasileira de Empresas de Pesquisa. Critério de classificação econômica Brasil: dados com base no levantamento sócio econômico 2000 IBOPE [online]; 2003. [acesso em: 23 jul. 2008]. Disponível em: http:/ /www.abep.org/novo/Content.aspx?ContentID=302
13. Nunes MF, Freire MCM. Qualidade de vida de cirurgiões-dentistas que atuam em um serviço público. Rev Saúde Pública. 2006;40(6):1019-26.

14. Saupe R, Nietche EA, Cestari ME, Giorgi MDM, Krahl M. Qualidade de vida dos acadêmicos de enfermagem. Rev Lat Am Enfermagem. 2004;12(4):636-42.

15. Raj SR, Simpson CS, Hopman WM, Singer MA. Health-related quality of life among final-year medical students. CMAJ. 2000;162(4):509-10.

16. Willcock SM, Daly MG, Tennant CC, Allard BJ. Burnout and psychiatric morbidity in new medical graduates. MJA. 2004;181(7):357-60.

17. Berlim MT, Fleck MPA. "Quality of life": a brand new concept for research and practice in psyquiatry. Rev Bras Psiquiatr. 2003;25(4):249-52.

18. Pereira RJ, Cotta RMM, Francheschini SCC, Ribeiro RCL, Sampaio RF, Priore SE, et al. Contribuição dos domínios físico, social, psicológico e ambiental para a qualidade de vida global de idosos. Rev Psiquiatr Rio Gd. Sul. 2006;28(1):27-38.

19. Roque VMN, Forones NM. Avaliação da qualidade de vida e toxicidade em pacientes com câncer colorretal tratados com quimioterapia adjuvante baseada em fluiropirimidinas. Arq Gastroenterol. 2006;43(2):94-101.

20. Parkerson GR Jr, Broadhead WE, Tse CK. The health status and life satisfaction of first-year medical students. Acad Med. 1990;65(9):586-8.

21. Ball S, Bax A. Self-care in medical education: effectiveness of health-habits interventions for first-year medical students. Acad Med. 2002;77(9):911-7.

22. Dyrbye LN, Thomas MR, Huntington JL, Lawson KL , Novotny PJ, Sloan JA, et al. Personal Life events and medical student burnout: a multicenter study. Acad Med. 2006;81(4):374-84.

23. Dyrbye LN, Thomas MR, Huschka MM, Lawson KL, Novotny PJ, Sloan JA, et al. A multicenter study of burnout, depression, and quality of life in minority and nonminority US medical students. Mayo Clin Proc. 2006;81(11):1435-42.

24. Dyrbye LN, Thomas MR, Shanafelt TD. Systematic review of depression, anxiety and others indicators of psychological distress among US and Canadian medical students. Acad Med. 2006;81(4):354-73.

25. Torres BS, Godoy I. Diretrizes para cessação do tabagismo. Doenças tabaco-relacionadas. J Bras Pneumol. 2004;30(supl.2):S19-29. 
26. Andrade APA, Bernardo ACC, Viegas CAA, Ferreira DBL, Gomes TC, Sales MR. Prevalência e características do tabagismo em jovens da Universidade de Brasília. J Bras Pneumol. 2006;32(1):23-8

27. Rondina RC, Moratelli HB, Botelho C. Tabagismo e características da personalidade em estudantes universitários. Rev Psiquiatr Clín [periódico na Internet]. 2001 [acesso em: 23 jul. 2008];28(2): [aproximadamente 10p.]. Disponível em: http://www.hcnet.usp.br/ipq/revista

28. Souza DPO, Areco KN, Silveira Filho DX. Álcool e alcoolismo entre adolescentes da rede estadual de ensino de Cuiabá, Mato Grosso. Rev Saúde Pública. 2005;39(4):585-92.

Apoio: Este trabalho foi realizado com o apoio do Centro de Bioética do Conselho Regional de Medicina do Estado de São Paulo (Cremesp).

\section{CONTRIBUIÇÃO DOS AUTORES}

João Carlos Ramos-Dias e Maria Helena Senger contribuíram na coordenação, análise dos resultados e redação do manuscrito. Milena Carvalho Libardi, Carina Maria Zillo e Mamy Honda Igarashi participaram na coleta de dados e na análise dos resultados.

\section{CONFLITO DE INTERESSES}

Declarou não haver.

\section{ENDEREÇO PARA CORRESPONDÊNCIA}

João Carlos Ramos-Dias

Rua da Paz, 535 - Chácara Santo Antônio

Alto da Boa Vista - São Paulo

CEP. 04713-000 SP

E-mail: jcrdias@pucsp.br 